\title{
Lacunary Statistical Limit and Cluster Points of Generalized Difference Sequences of Fuzzy Numbers
}

\author{
Pankaj Kumar, ${ }^{1}$ Vijay Kumar, ${ }^{1}$ and S. S. Bhatia ${ }^{2}$ \\ ${ }^{1}$ Department of Mathematics, Haryana College of Technology and Management, Haryana, Kaithal 136027, India \\ ${ }^{2}$ School of Mathematics and Computer Application, Thapar University, Punjab, Patiala 147004, India
}

Correspondence should be addressed to Pankaj Kumar, pankaj.lankesh@yahoo.com

Received 20 April 2012; Accepted 14 June 2012

Academic Editor: Katsuhiro Honda

Copyright ( 2012 Pankaj Kumar et al. This is an open access article distributed under the Creative Commons Attribution License, which permits unrestricted use, distribution, and reproduction in any medium, provided the original work is properly cited.

The aim of present work is to introduce and study lacunary statistical limit and lacunary statistical cluster points for generalized difference sequences of fuzzy numbers. Some inclusion relations among the sets of ordinary limit points, statistical limit points, statistical cluster points, lacunary statistical limit points, and lacunary statistical cluster points for these type of sequences are obtained.

\section{Introduction}

The notion of statistical convergence of sequences of numbers was introduced by Fast [1] and Schoenberg [2] independently and latter discussed in [3-6], and so forth. In 1993, Fridy and Orhan [7] presented an interesting generalization of statistical convergence with the help of a lacunary sequence and called it lacunary statistical convergence or $S_{\theta^{-}}$ convergence. Demirci [8] defined $S_{\theta}$-limit and cluster points of number sequences and obtained some interesting results analogous to [4]. In past years, statistical convergence has also become an interesting area of research for sequences of fuzzy numbers. The credit goes to Nuray and Savaş [9] who first introduced statistical convergence of sequences of fuzzy numbers. After their pioneer work, many authors have made their contribution to study different generalizations of statistical convergence for sequences of fuzzy numbers (see [10-13], etc.).

Quite recently, statistical convergence of sequences of fuzzy numbers is studied with the help of the difference operator $\Delta$. For instance, Bilgin [14] introduced strongly $\Delta$-summable and $\Delta$-statistical convergence of sequences of fuzzy numbers. Işik [15] studied some notions of generalized difference sequences of numbers. In 2006, Altin et al. [16] united lacunary sequences to introduce the concept of lacunary statistical convergence of generalized difference sequences of fuzzy numbers and obtained some interesting results. Some more work in this direction can be found in [17-19]. In present work, we continue with this study and introduce the concepts of lacunary statistical limit and cluster points of generalized difference sequences of fuzzy numbers. We obtain some relations among the sets of ordinary limit, points, lacunary statistical limit, and cluster points for these type of sequences.

\section{Background and Preliminaries}

We begin with the following terminology on fuzzy numbers. Given any interval $A$, we shall denote its end points by $\underline{A}, \bar{A}$ and by $D$ the set of all closed bounded intervals on real line $\mathbb{R}$, that is, $D=\{A \subset \mathbb{R}: A=[\underline{A}, \bar{A}]\}$. For $A, B \in D$ we define $A \leq B$ if and only if $A \leq B$ and $\bar{A} \leq \bar{B}$. Moreover, the distance function $d$ defined by $d(A, B)=\max \{|\underline{A}-\underline{B}|,|\bar{A}-\bar{B}|\}$ is a Hausdorff metric on $D$ and $(D, d)$ is a complete metric space. Also $\leq$ is a partial order on $D$.

A fuzzy number is a function $X$ from $\mathbb{R}$ to $[0,1]$ which is satisfying the following conditions: (i) $X$ is normal, that is, there exists $x_{0} \in \mathbb{R}$ such that $X\left(x_{0}\right)=1$; (ii) $X$ is fuzzy convex, that is, for any $x, y \in \mathbb{R}$ and $\lambda \in[0,1], X(\lambda x+(1-$ d) $y) \geq \min \{X(x), X(y)\}$; (iii) $X$ is upper semicontinuous; and (iv) the closure of the set $\{x \in \mathbb{R}: X(x)>0\}$ denoted by $X^{0}$ is compact. 
Properties (i)-(iv) imply that for each $\alpha \in(0,1]$, the $\alpha$-level set, $X^{\alpha}=\{x \in \mathbb{R}: X(x) \geq \alpha\}=\left[\underline{X}^{\alpha}, \bar{X}^{\alpha}\right]$, is a nonempty compact convex subset of $\mathbb{R}$. Let $L(\mathbb{R})$ denote the set of all fuzzy numbers. The linear structure of $L(\mathbb{R})$ induces an addition $X+Y$ and a scalar multiplication $\lambda X$ in terms of $\alpha$-level sets by

$$
\begin{gathered}
{[X+Y]^{\alpha}=[X]^{\alpha}+[Y]^{\alpha},} \\
{[\lambda X]^{\alpha}=\lambda[X]^{\alpha} \quad(X, Y \in L(\mathbb{R}), \lambda \in \mathbb{R})}
\end{gathered}
$$

for each $\alpha \in[0,1]$. Define a map $\bar{d}: L(\mathbb{R}) \times L(\mathbb{R}) \rightarrow \mathbb{R}$ by

$$
\bar{d}(X, Y)=\sup _{\alpha \in[0,1]} d\left(X^{\alpha}, Y^{\alpha}\right) .
$$

Puri and Ralescu [20] proved that $(L(\mathbb{R}), \bar{d})$ is a complete metric space. Also the ordered structure on $L(\mathbb{R})$ is defined as follows. For $X, Y \in L(\mathbb{R})$, we define $X \leq Y$ if and only if $\underline{X}^{\alpha} \leq \underline{Y}^{\alpha}$ and $\bar{X}^{\alpha} \leq \bar{Y}^{\alpha}$ for each $\alpha \in[0,1]$. We say that $X<Y$ if $X \leq Y$ and there exist $\alpha_{0} \in[0,1]$ such that $\underline{X}^{\alpha_{0}}<\underline{Y}^{\alpha_{0}}$ or $\bar{X}^{\alpha_{0}}<\bar{Y}^{\alpha_{0}}$. The fuzzy numbers $X$ and $Y$ are said to be incomparable if neither $X \leq Y$ nor $Y \leq X$.

We next recall some definitions and results which form the base for present study. For any set $K \subseteq \mathbb{N}$, let $K_{n}$ denote the set $\{k \in K: k \leq n\}$ and $\left|K_{n}\right|$ denote the number of elements in $K_{n}$. The natural density $\delta$ of $K$ is defined by $\delta(K)=\lim _{n} n^{-1}\left|K_{n}\right|$. The natural density may not exist for each set $K$. But the upper density $\bar{\delta}$ defined by $\bar{\delta}(K)=$ $\lim \sup _{n} n^{-1}\left|K_{n}\right|$ always exists for each set $K$. Moreover, $\delta(K)$ different from zero means $\bar{\delta}(K)>0$. Besides that, $\delta\left(K^{C}\right)=$ $1-\delta(K)$ and if $A \subseteq B$, then $\bar{\delta}(A) \leq \bar{\delta}(B)$.

For any sequence $X=\left(X_{k}\right)$ of fuzzy numbers, we write $\left\{X_{k}: k \in \mathbb{N}\right\}$ to denote the range of $X$. If $\left(X_{k(j)}\right)$ is a subsequence of $X$ and $K=\{k(j): j \in \mathbb{N}\}$, then we abbreviate $\left(X_{k(j)}\right)$ by $(X)_{K}$. If $\delta(K)=0,(X)_{K}$ is called a thin subsequence, otherwise if $\delta(K) \neq 0,(X)_{K}$ is called nonthin subsequence of $X$.

For $w$, the set of all sequences of fuzzy numbers, the operator $\Delta^{m}: w \rightarrow w$ is defined by

$$
\begin{aligned}
\Delta^{0} X_{k} & =X_{k}, \\
\Delta^{1} X_{k} & =X_{k}-X_{k+1}, \\
\vdots & =\vdots \\
\Delta^{m} X_{k} & =\Delta^{1}\left(\Delta^{m-1} X_{k}\right) .
\end{aligned}
$$

Definition 1. A sequence $X=\left(X_{k}\right)$ of fuzzy numbers is said to be $\Delta^{m}$-statistically convergent to a fuzzy number $X_{0}$, in symbol: $S\left(\Delta^{m}\right)-\lim _{k} X_{k}=X_{0}$, if for each $\epsilon>0$,

$$
\lim _{n \rightarrow \infty} \frac{1}{n}\left|\left\{k \in \mathbb{N}: \bar{d}\left(\Delta^{m} X_{k}, X_{0}\right) \geq \epsilon\right\}\right|=0 .
$$

Let $S\left(\Delta^{m}(X)\right)$ denote the set of all $\Delta^{m}$-statistically convergent sequences of fuzzy numbers.

Definition 2. Let $X=\left(X_{k}\right)$ be a sequence of fuzzy numbers. A fuzzy number $X_{0}$ is said to be a statistical limit point (s.l.p) of the generalized difference sequence $\left(\Delta^{m} X_{k}\right)$ of fuzzy numbers provided that there is a nonthin subsequence of $X$ that is $\Delta^{m}$ convergent to $X_{0}$.

Let $\Lambda_{S}\left(\Delta^{m}(X)\right)$ denote the set of all s.l.p. of the generalized difference sequence $\left(\Delta^{m} X_{k}\right)$ of fuzzy numbers.

Definition 3. Let $X=\left(X_{k}\right)$ be a sequence of fuzzy numbers. A fuzzy number $Y_{0}$ is said to be a statistical cluster point (s.c.p) of the generalized difference sequence $\left(\Delta^{m} X_{k}\right)$ of fuzzy numbers provided that, for each $\epsilon>0$,

$$
\limsup _{n \rightarrow \infty} \frac{1}{n}\left|\left\{k \in \mathbb{N}: \bar{d}\left(\Delta^{m} X_{k}, Y_{0}\right)<\epsilon\right\}\right|>0
$$

Let $\Gamma_{S}\left(\Delta^{m}(X)\right)$ denote the set of all s.c.p of the generalized difference sequence $\left(\Delta^{m} X_{k}\right)$ of fuzzy numbers.

By a lacunary sequence we mean an increasing sequence $\theta=\left(k_{r}\right)$ of positive integers such that $k_{0}=0$ and $h_{r}=k_{r}-$ $k_{r-1} \rightarrow \infty$ as $r \rightarrow \infty$. The intervals determined by $\theta=\left(k_{r}\right)$ will be denoted by $I_{r}=\left(k_{r-1}, k_{r}\right]$ whereas the ratio $k_{r} / k_{r-1}$ is denoted by $q_{r}$. Further, a lacunary sequence $\theta^{\prime}=\left(k_{r}^{\prime}\right)$ is called a lacunary refinement of the lacunary sequence $\theta=$ $\left(k_{r}\right)$ if $\left\{k_{r}\right\} \subseteq\left\{k_{r}^{\prime}\right\}$.

Definition 4 (see [21]). Let $\theta=\left(k_{r}\right)$ be a lacunary sequence. A sequence $X=\left(X_{k}\right)$ of fuzzy numbers is said to be lacunary statistical convergent to a fuzzy number $X_{0}$ provided that for each $\epsilon>0$,

$$
\lim _{r \rightarrow \infty} \frac{1}{h_{r}}\left|\left\{k \in I_{r}: \bar{d}\left(X_{k}, Y_{0}\right) \geq \epsilon\right\}\right|=0 .
$$

Let $S_{\theta}$ denote the set of all lacunary statistically convergent sequences of fuzzy numbers.

Let $\theta=\left(k_{r}\right)$ be a lacunary sequence and $X=\left(X_{k}\right)$ a sequence of fuzzy numbers. If $(X)_{K}$ where $K=\{k(j): j \in$ $\mathbb{N}$ \} is a subsequence of $X=\left(X_{k}\right)$ such that

$$
\lim _{r \rightarrow \infty} \frac{1}{h_{r}}\left|\left\{k(j) \in I_{r}: j \in \mathbb{N}\right\}\right|=0,
$$

we call $(X)_{K}$ a $\theta$-thin subsequence. On the other hand, $(X)_{K}$ is a $\theta$-nonthin subsequence of $X$ provided that

$$
\limsup _{r \rightarrow \infty} \frac{1}{h_{r}}\left|\left\{k(j) \in I_{r}: j \in \mathbb{N}\right\}\right|>0 .
$$

Definition 5. Let $\theta=\left(k_{r}\right)$ be a lacunary sequence. A sequence $X=\left(X_{k}\right)$ of fuzzy numbers is said to be lacunary $\Delta^{m_{-}}$ statistically convergent to a fuzzy number $X_{0}$, in symbol: $S_{\theta}\left(\Delta^{m}\right)-\lim _{k} X_{k}=X_{0}$, if for each $\epsilon>0$,

$$
\lim _{r \rightarrow \infty} \frac{1}{h_{r}}\left|\left\{k \in I_{r}: \bar{d}\left(\Delta^{m} X_{k}, X_{0}\right) \geq \epsilon\right\}\right|=0 .
$$

Let $S_{\theta}\left(\Delta^{m}(X)\right)$ denote the set of all lacunary $\Delta^{m}$-statistically convergent sequences of fuzzy numbers.

We now consider the natural definitions of statistical limit and cluster points for generalized difference sequences of fuzzy numbers with respect to lacunary sequences. 


\section{Main Results}

Definition 6. Let $\theta=\left(k_{r}\right)$ be a lacunary sequence and $X=$ $\left(X_{k}\right)$ a sequence of fuzzy numbers. A fuzzy number $X_{0}$ is said to be a lacunary statistical limit point (l.s.l.p) of the generalized difference sequence $\left(\Delta^{m} X_{k}\right)$ of fuzzy numbers provided that there is a $\theta$-nonthin subsequence of $X$ that is $\Delta^{m}$-convergent to $X_{0}$.

Let $\Lambda_{S_{\theta}}\left(\Delta^{m}(X)\right)$ denote the set of all 1.s.l.p. of the generalized difference sequence $\left(\Delta^{m} X_{k}\right)$ of fuzzy numbers.

Definition 7. Let $\theta=\left(k_{\mathrm{r}}\right)$ be a lacunary sequence and $X=$ $\left(X_{k}\right)$ a sequence of fuzzy numbers. A fuzzy number $Y_{0}$ is said to be a lacunary statistical cluster point (l.s.c.p) of the generalized difference sequence $\left(\Delta^{m} X_{k}\right)$ of fuzzy numbers provided that, for each $\epsilon>0$,

$$
\limsup _{r \rightarrow \infty} \frac{1}{h_{r}}\left|\left\{k \in I_{r}: \bar{d}\left(\Delta^{m} X_{k}, Y_{0}\right)<\epsilon\right\}\right|>0 .
$$

Let $\Gamma_{S_{\theta}}\left(\Delta^{m}(X)\right)$ denote the set of all 1.s.c.p of the generalized difference sequence $\left(\Delta^{m} X_{k}\right)$ of fuzzy numbers.

Example 8. Let $\theta=\left(k_{r}\right)$ be a lacunary sequence. We define a sequence of fuzzy numbers $X=\left(X_{k}\right)$ as follows. For $x \in \mathbb{R}$, define

$$
X_{k}(x)=\left\{\begin{array}{cc}
x-k+1, & \text { if } k-1 \leq x \leq k \\
-x+k+1, & \text { if } k<x \leq k+1 \\
0, & \text { otherwise }
\end{array}\right\},
$$

Then, we obtain

$$
\begin{aligned}
& {\left[X_{k}\right]^{\alpha}= \begin{cases}{[k-1+\alpha, k+1-\alpha],} & \text { if } k \in\left[k_{r}-\left[\sqrt{h_{r}}\right]+1, k_{r}\right] \\
{[5+\alpha, 7-\alpha]} & \text { otherwise, }\end{cases} } \\
& {\left[\Delta X_{k}\right]^{\alpha}=\left\{\begin{array}{l}
{[-3+\alpha, 1-2 \alpha],} \\
\quad \text { if both } k, k+1 \in\left[k_{r}-\left[\sqrt{h_{r}}\right]+1, k_{r}\right] \\
\quad \text { if } k \in\left[k_{r}-\left[\sqrt{h_{r}}\right]+1, k_{r}\right] \text { but not } k+1, \\
{[-k+3+2 \alpha,-k+7-2 \alpha],} \\
\quad \text { if } k+1 \in\left[k_{r}-\left[\sqrt{h_{r}}\right]+1, k_{r}\right] \text { but not } k, \\
{[-2+2 \alpha, 2-2 \alpha], \quad \text { otherwise. }}
\end{array}\right.}
\end{aligned}
$$

Thus, for $m=1$, it is clear that the sequence $\Delta X_{k}$ has two different subsequences which converge to $\mu_{1}$ and $\mu_{2}$, respectively, where $\left[\mu_{1}\right]^{\alpha}=[-3+\alpha, 1-2 \alpha]$ and $\left[\mu_{2}\right]^{\alpha}=[-2+$ $2 \alpha, 2-2 \alpha]$. Hence, if $L(\Delta(X))$ denotes the set of ordinary limit points of $\left(\Delta X_{k}\right)$, then $L(\Delta(X))=\left\{\mu_{1}, \mu_{2}\right\}$; however, $\Gamma_{S_{\theta}}(\Delta(X))=\left\{\mu_{2}\right\}$.
Theorem 9. Let $\theta=\left(k_{r}\right)$ be a lacunary sequence and $X=\left(X_{k}\right)$ a sequence of fuzzy numbers. Then, one has $\Lambda_{S_{\theta}}\left(\Delta^{m}(X)\right) \subseteq \Gamma_{S_{\theta}}\left(\Delta^{m}(X)\right)$.

Proof. Suppose $X_{0} \in \Lambda_{S_{\theta}}\left(\Delta^{m}(X)\right)$. By definition, there is a $\theta$-nonthin subsequence $\left(X_{k(j)}\right)$ of $X=\left(X_{k}\right)$ which is $\Delta^{m_{-}}$ convergent to $X_{0}$, and therefore we have

$$
\limsup _{r \rightarrow \infty} \frac{1}{h_{r}}\left|\left\{k(j) \in I_{r}: j \in \mathbb{N}\right\}\right|=d>0 .
$$

Since, for every $\epsilon>0$,

$$
\left\{k \in I_{r}: \bar{d}\left(\Delta^{m} X_{k}, X_{0}\right)<\epsilon\right\} \supseteq\left\{k(j) \in I_{r}: \bar{d}\left(\Delta^{m} X_{k(j)}, X_{0}\right)<\epsilon\right\},
$$

so we have the containment

$$
\begin{aligned}
\{k \in & \left.I_{r}: \bar{d}\left(\Delta^{m} X_{k}, X_{0}\right)<\epsilon\right\} \\
\supset & \left\{k(j) \in I_{r}: j \in \mathbb{N}\right\} \\
& -\left\{k(j) \in I_{r}: \bar{d}\left(\Delta^{m} X_{k(j)}, X_{0}\right) \geq \epsilon\right\} .
\end{aligned}
$$

Now, $\left(X_{k(j)}\right)$ is $\Delta^{m}$-convergent to $X_{0}$, which implies that, for every $\epsilon>0,\left\{k(j) \in I_{r}: \bar{d}\left(\Delta^{m} X_{k(j)}, X_{0}\right) \geq \epsilon\right\}$ is finite for which we have

$$
\limsup _{r \rightarrow \infty} \frac{1}{h_{r}}\left|\left\{k(j) \in I_{r}: \bar{d}\left(\Delta^{m} X_{k(j)}, X_{0}\right) \geq \epsilon\right\}\right|=0 .
$$

Thus from (15), we obtain

$$
\begin{aligned}
\limsup _{r \rightarrow \infty} & \frac{1}{h_{r}}\left|\left\{k \in I_{r}: \bar{d}\left(\Delta^{m} X_{k}, X_{0}\right)<\epsilon\right\}\right| \\
\geq & \limsup _{r \rightarrow \infty} \frac{1}{h_{r}}\left|\left\{k(j) \in I_{r}: j \in \mathbb{N}\right\}\right| \\
& \quad-\limsup _{r \rightarrow \infty} \frac{1}{h_{r}}\left|\left\{k(j) \in I_{r}: \bar{d}\left(\Delta^{m} X_{k(j)}, X_{0}\right) \geq \epsilon\right\}\right| \\
\geq & \limsup _{r \rightarrow \infty} \frac{1}{h_{r}}\left|\left\{k(j) \in I_{r}: j \in \mathbb{N}\right\}\right|=d>0,
\end{aligned}
$$

using (13) and (16). This shows that $X_{0} \in \Gamma_{S_{\theta}}\left(\Delta^{m}(X)\right)$ and therefore the result is proved.

Theorem 10. Let $\theta=\left(k_{r}\right)$ be a lacunary sequence. Then, for any sequence $X=\left(X_{k}\right)$ of fuzzy numbers, one has $\Gamma_{S_{\theta}}\left(\Delta^{m}(X)\right) \subseteq L\left(\Delta^{m}(X)\right)$.

Proof. Assume $Y_{0} \in \Gamma_{S_{\theta}}\left(\Delta^{m}(X)\right)$. By definition, for each $\epsilon>$ 0 we have

$$
\limsup _{r \rightarrow \infty} \frac{1}{h_{r}}\left|\left\{k \in I_{r}: \bar{d}\left(\Delta^{m} X_{k}, Y_{0}\right)<\epsilon\right\}\right|>0 .
$$

We set $(X)_{K}$ a $\theta$-nonthin subsequence of $X=\left(X_{k}\right)$ such that $K=\left\{k(j) \in I_{r}: \bar{d}\left(\Delta^{m} X_{k}, Y_{0}\right)<\epsilon\right\}$ for $\epsilon>0$. Since $\lim \sup _{r \rightarrow \infty}\left(1 / h_{r}\right)|\{K\}|>0$, it follows that $K$ is an infinite set. Thus we have a subsequence $(X)_{K}$ of $X$ that is $\Delta^{m}$-convergent to $Y_{0}$. This shows that $Y_{0} \in L\left(\Delta^{m}(X)\right)$. Hence $\Gamma_{S_{\theta}}\left(\Delta^{m}(X)\right) \subseteq L\left(\Delta^{m}(X)\right)$. 
Theorem 11. Let $\theta=\left(k_{r}\right)$ be a lacunary sequence. If $X=\left(X_{k}\right)$ and $Y=\left(Y_{k}\right)$ are two sequences of fuzzy numbers such that $\lim _{r \rightarrow \infty}\left(1 / h_{r}\right)\left|\left\{k \in I_{r}: X_{k} \neq Y_{k}\right\}\right|=0$, then $\Lambda_{S_{\theta}}\left(\Delta^{m}(X)\right)=$ $\Lambda_{S_{\theta}}\left(\Delta^{m}(Y)\right)$ and $\Gamma_{S_{\theta}}\left(\Delta^{m}(X)\right)=\Gamma_{S_{\theta}}\left(\Delta^{m}(Y)\right)$.

Proof. We prove the theorem into two parts. In the first part we prove that $\Lambda_{S_{\theta}}\left(\Delta^{m}(X)\right)=\Lambda_{S_{\theta}}\left(\Delta^{m}(Y)\right)$; however, in the second part we shall prove $\Gamma_{S_{\theta}}\left(\Delta^{m}(X)\right)=\Gamma_{S_{\theta}}\left(\Delta^{m}(Y)\right)$.

Part (i). Let $X_{0} \in \Lambda_{S_{\theta}}\left(\Delta^{m}(Y)\right)$. By definition, there is a $\theta$-nonthin subsequence $(Y)_{K}$ of $Y=\left(Y_{k}\right)$ that is $\Delta^{m_{-}}$ convergent to $X_{0}$. Since $\lim _{r \rightarrow \infty}\left(1 / h_{r}\right) \mid\left\{k \in I_{r}: k \in\right.$ $K$ and $\left.X_{k} \neq Y_{k}\right\} \mid=0$, it follows that $\lim \sup _{r \rightarrow \infty}\left(1 / h_{r}\right) \mid\{k \in$ $I_{r}: k \in K$ and $\left.X_{k}=Y_{k}\right\} \mid>0$. Therefore, from the later set, we can yield a $\theta$-nonthin subsequence $(X)_{K^{\prime}}$ of $X=$ $\left(X_{k}\right)$ that is $\Delta^{m}$-convergent to $X_{0}$. Hence, $X_{0} \in \Lambda_{S_{\theta}}\left(\Delta^{m}(X)\right)$, and therefore we have $\Lambda_{S_{\theta}}\left(\Delta^{m}(Y)\right) \subset \Lambda_{S_{\theta}}\left(\Delta^{m}(X)\right)$. Also by symmetry one get $\Lambda_{S_{\theta}}\left(\Delta^{m}(X)\right) \subset \Lambda_{S_{\theta}}\left(\Delta^{m}(Y)\right)$. On combining we have $\Lambda_{S_{\theta}}\left(\Delta^{m}(X)\right)=\Lambda_{S_{\theta}}\left(\Delta^{m}(Y)\right)$.

Part (ii). Let $Y_{0} \in \Gamma_{S_{\theta}}\left(\Delta^{m}(X)\right)$. By definition, for each $\epsilon>0$,

$$
\limsup _{r \rightarrow \infty} \frac{1}{h_{r}}\left|\left\{k \in I_{r}: \bar{d}\left(\Delta^{m} X_{k}, Y_{0}\right)<\epsilon\right\}\right|>0 .
$$

Since $X_{k}=Y_{k}$ for all most all $k$, it follows that, for each $\epsilon>0$,

$$
\limsup _{r \rightarrow \infty} \frac{1}{h_{r}}\left|\left\{k \in I_{r}: \bar{d}\left(\Delta^{m} Y_{k}, Y_{0}\right)<\epsilon\right\}\right|>0
$$

This shows that $Y_{0} \in \Gamma_{S_{\theta}}\left(\Delta^{m}(Y)\right)$ and therefore $\Gamma_{S_{\theta}}\left(\Delta^{m}(X)\right) \subset \Gamma_{S_{\theta}}\left(\Delta^{m}(Y)\right)$. By symmetry, we see that $\Gamma_{S_{\theta}}\left(\Delta^{m}(Y)\right) \subset \Gamma_{S_{\theta}}\left(\Delta^{m}(X)\right)$, whence $\Gamma_{S_{\theta}}\left(\Delta^{m}(X)\right)=$ $\Gamma_{S_{\theta}}\left(\Delta^{m}(Y)\right)$.

Theorem 12. Let $\theta=\left(k_{r}\right)$ be a lacunary sequence. If $X=\left(X_{k}\right)$ is a sequence of fuzzy numbers such that $S_{\theta}\left(\Delta^{m}\right)-\lim _{k} X_{k}=$ $X_{0}$, then $\Lambda_{S_{\theta}}\left(\Delta^{m}(X)\right)=\Gamma_{S_{\theta}}\left(\Delta^{m}(X)\right)=\left\{X_{0}\right\}$.

Proof. We prove the theorem in two parts. In the first part, we prove that $\Lambda_{S_{\theta}}\left(\Delta^{m}(X)\right)=\left\{X_{0}\right\}$ whereas in the second part we obtain $\Gamma_{S_{\theta}}\left(\Delta^{m}(X)\right)=\left\{X_{0}\right\}$.

Part (i). Suppose that $\Lambda_{S_{\theta}}\left(\Delta^{m}(X)\right)=\left\{X_{0}, Y_{0}\right\}$, where $X_{0} \neq Y_{0}$, that is, $Y_{0}$ is a l.s.l.p. of the generalized difference sequence $\left(\Delta^{m} X_{k}\right)$ different from $X_{0}$. Choose $\epsilon>0$ such that $0<\epsilon<\bar{d}\left(X_{0}, Y_{0}\right) / 2$. By definition there exist two $\theta$-nonthin subsequences $\left(X_{k(j)}\right)$ and $\left(X_{l(i)}\right)$ of the sequence $X=\left(X_{k}\right)$ which are $\Delta^{m}$-convergent to $X_{0}$ and $Y_{0}$, respectively. Since $\left(X_{l(i)}\right)$ is $\Delta^{m}$-convergent to $Y_{0}$, so for each $\epsilon>0,\left\{l(i) \in I_{n}\right.$ : $\left.\bar{d}\left(\Delta^{m} X_{l(i)}, Y_{0}\right) \geq \epsilon\right\}$ is a finite set for which

$$
\limsup _{r \rightarrow \infty} \frac{1}{h_{r}}\left|\left\{l(i) \in I_{r}: \bar{d}\left(\Delta^{m} X_{l(i)}, Y_{0}\right) \geq \epsilon\right\}\right|=0 .
$$

Further, we can write

$$
\begin{aligned}
\left\{l(i) \in I_{r}: i \in \mathbb{N}\right\}= & \left\{l(i) \in I_{r}: \bar{d}\left(\Delta^{m} X_{l(i)}, Y_{0}\right)<\epsilon\right\} \\
& \cup\left\{l(i) \in I_{r}: \bar{d}\left(\Delta^{m} X_{l(i)}, Y_{0}\right) \geq \epsilon\right\},
\end{aligned}
$$

for which we have

$$
\begin{aligned}
\limsup _{r \rightarrow \infty} & \frac{1}{h_{r}}\left|\left\{l(i) \in I_{r}: i \in \mathbb{N}\right\}\right| \\
= & \limsup _{r \rightarrow \infty} \frac{1}{h_{r}}\left|\left\{l(i) \in I_{r}: \bar{d}\left(\Delta^{m} X_{l(i)}, Y_{0}\right)<\epsilon\right\}\right| \\
& +\limsup _{r \rightarrow \infty} \frac{1}{h_{r}}\left|\left\{l(i) \in I_{r}: \bar{d}\left(\Delta^{m} X_{l(i)}, Y_{0}\right) \geq \epsilon\right\}\right| .
\end{aligned}
$$

Since $\left(X_{l(i)}\right)$ is nonthin, so, by use of $(21)$, we have

$$
\limsup _{r \rightarrow \infty} \frac{1}{h_{r}}\left|\left\{l(i) \in I_{r}: \bar{d}\left(\Delta^{m} X_{l(i)}, Y_{0}\right)<\epsilon\right\}\right|>0 .
$$

Since $S_{\theta}\left(\Delta^{m}\right)-\lim _{k} X_{k}=X_{0}$, so for each $\epsilon>0$

$$
\lim _{r \rightarrow \infty} \frac{1}{h_{r}}\left|\left\{k \in I_{r}: \bar{d}\left(\Delta^{m} X_{k}, X_{0}\right) \geq \epsilon\right\}\right|=0,
$$

and therefore we can write

$$
\limsup _{r \rightarrow \infty} \frac{1}{h_{r}}\left|\left\{k \in I_{r}: \bar{d}\left(\Delta^{m} X_{k}, X_{0}\right)<\epsilon\right\}\right|>0
$$

Furthermore for $0<2 \epsilon<\bar{d}\left(X_{0}, Y_{0}\right)$,

$$
\begin{aligned}
\{l(i) & \left.\in I_{r}: \bar{d}\left(\Delta^{m} X_{l(i)}, Y_{0}\right)<\epsilon\right\} \\
& \cap\left\{k \in I_{r}: \bar{d}\left(\Delta^{m} X_{k}, X_{0}\right)<\epsilon\right\}=\varnothing,
\end{aligned}
$$

which immediately gives the containment

$$
\left\{l(i) \in I_{r}: \bar{d}\left(\Delta^{m} X_{l(i)}, Y_{0}\right)<\epsilon\right\} \subset\left\{k \in I_{r}: \bar{d}\left(\Delta^{m} X_{k}, X_{0}\right) \geq \epsilon\right\}
$$

for which we have

$$
\begin{aligned}
& \limsup _{r \rightarrow \infty} \frac{1}{h_{r}}\left|\left\{l(i) \in I_{r}: \bar{d}\left(\Delta^{m} X_{l(i)}, Y_{0}\right)<\epsilon\right\}\right| \\
& \quad \leq \limsup _{r \rightarrow \infty} \frac{1}{h_{r}}\left|\left\{k \in I_{r}: \bar{d}\left(\Delta^{m} X_{k}, X_{0}\right) \geq \epsilon\right\}\right|=0 .
\end{aligned}
$$

As left side of (29) cannot be negative, so we must have

$$
\limsup _{r \rightarrow \infty} \frac{1}{h_{r}}\left|\left\{l(i) \in I_{r}: \bar{d}\left(\Delta^{m} X_{l(i)}, Y_{0}\right)<\epsilon\right\}\right|=0 .
$$

This contradicts (24). Hence, $\Lambda_{S_{\theta}}\left(\Delta^{m}(X)\right)=\left\{X_{0}\right\}$.

Part (ii). Let $Z_{0}$ be a l.s.c.p. of the generalized difference sequence $\left(\Delta^{m} X_{k}\right)$ different from $X_{0}$, that is, $\Gamma_{S_{\theta}}\left(\Delta^{m}(X)\right)=$ $\left\{X_{0}, Z_{0}\right\}$, where $X_{0} \neq Z_{0}$. Choose $\epsilon$ such that $0<\epsilon<$ $\bar{d}\left(X_{0}, Z_{0}\right) / 2$. Since $Z_{0}$ is a l.s.c.p of $\left(\Delta^{m} X_{k}\right)$, so for each $\epsilon>0$ we have

$$
\limsup _{r \rightarrow \infty} \frac{1}{h_{r}}\left|\left\{k \in I_{r}: \bar{d}\left(\Delta^{m} X_{k}, Z_{0}\right)<\epsilon\right\}\right|>0 .
$$

Since $\left\{k \in I_{r}: \bar{d}\left(\Delta^{m} X_{k}, X_{0}\right)<\epsilon\right\} \cap\left\{k \in I_{r}: \bar{d}\left(\Delta^{m} X_{k}, Z_{0}\right)<\right.$ $\epsilon\}=\varnothing$ for every $0<\epsilon<d\left(X_{0}, Z_{0}\right) / 2$, it follows that 
$\left\{k \in I_{r}: \bar{d}\left(\Delta^{m} X_{k}, X_{0}\right) \geq \epsilon\right\} \supseteq\left\{k \in I_{r}: \bar{d}\left(\Delta^{m} X_{k}, Z_{0}\right)<\epsilon\right\}$ for which we have

$$
\begin{aligned}
& \limsup _{r \rightarrow \infty} \frac{1}{h_{r}}\left|\left\{k \in I_{r}: \bar{d}\left(\Delta^{m} X_{k}, X_{0}\right) \geq \epsilon\right\}\right| \\
& \quad \geq \limsup _{r \rightarrow \infty} \frac{1}{h_{r}}\left|\left\{k \in I_{r}: \bar{d}\left(\Delta^{m} X_{k}, Z_{0}\right)<\epsilon\right\}\right|>0
\end{aligned}
$$

by (31), which is impossible as by (25) $\lim \sup _{r \rightarrow \infty}\left(1 / h_{r}\right)\left|\left\{k \in I_{r}: \bar{d}\left(\Delta^{m} X_{k}, X_{0}\right) \geq \epsilon\right\}\right|=0$. In this way we obtained a contradiction. Hence, $\Gamma_{S_{\theta}}\left(\Delta^{m}(X)\right)=\left\{X_{0}\right\}$.

Theorem 13. Let $\theta=\left(k_{r}\right)$ be a lacunary sequence and $X=$ $\left(X_{k}\right)$ a sequence of fuzzy numbers. Then one has the following:

(i) if $\lim \inf q_{r}>1$, then $\Lambda_{S_{\theta}}\left(\Delta^{m}(X)\right) \subset \Lambda_{S}\left(\Delta^{m}(X)\right)$,

(ii) if $\lim \sup q_{r}<\infty$, then $\Lambda_{S}\left(\Delta^{m}(X)\right) \subset \Lambda_{S_{\theta}}\left(\Delta^{m}(X)\right)$,

(iii) if $1<\lim \inf q_{r} \leq \lim \sup q_{r}<\infty$, then $\Lambda_{S}\left(\Delta^{m}(X)\right)=\Lambda_{S_{\theta}}\left(\Delta^{m}(X)\right)$.

Proof. (i) Suppose $\lim \inf q_{r}>1$; there exists a $\delta>0$ such that $q_{r}>1+\delta$ for sufficient large $r$, which implies that $h_{r} / k_{r} \geq$ $\delta /(1+\delta)$. Assume that $X_{0} \in \Lambda_{S_{\theta}}\left(\Delta^{m}(X)\right)$, then there is $\theta$ nonthin subsequence $\left(X_{k(j)}\right)$ of $\left(X_{k}\right)$ that is $\Delta^{m}$-convergent to $X_{0}$ and

$$
\limsup _{r \rightarrow \infty} \frac{1}{h_{r}}\left|\left\{k(j) \in I_{r}: j \in \mathbb{N}\right\}\right|=d>0 .
$$

Since

$$
\begin{aligned}
\frac{1}{k_{r}}\left|\left\{k(j) \leq k_{r}: j \in \mathbb{N}\right\}\right| \\
\geq \frac{1}{k_{r}}\left|\left\{k(j) \in I_{r}: j \in \mathbb{N}\right\}\right| \\
\quad=\frac{h_{r}}{k_{r}} \frac{1}{h_{r}}\left|\left\{k(j) \in I_{r}: j \in \mathbb{N}\right\}\right| \\
\geq\left(\frac{\delta}{1+\delta}\right) \frac{1}{h_{r}}\left|\left\{k(j) \in I_{r}: j \in \mathbb{N}\right\}\right|,
\end{aligned}
$$

it follows by (33) that $\lim \sup _{r \rightarrow \infty}\left(1 / k_{r}\right) \mid\left\{k(j) \leq k_{r}: j \in\right.$ $\mathbb{N}\} \mid>0$. Since $\left(X_{k(j)}\right)$ is already $\Delta^{m}$-convergent to $X_{0}$, so we have $X_{0} \in \Lambda_{S}\left(\Delta^{m}(X)\right)$. Hence $\Lambda_{S_{\theta}}\left(\Delta^{m}(X)\right) \subset \Lambda_{S}\left(\Delta^{m}(X)\right)$.

(ii) If $\lim \sup q_{r}<\infty$, then there exists a real number $H$ such that $q_{r}<H$ for all $r$. Without loss of generality, we can assume $H>1$ (as otherwise $k_{r}<k_{r-1}$ ). Now for all $r,\left(h_{r} / k_{r-1}\right)=\left(k_{r}-k_{r-1}\right) / k_{r-1}=q_{r}-1 \leq$ $H-1$. Let $X_{0} \in \Lambda_{S}\left(\Delta^{m}(X)\right)$, then there is a set $K=$ $\{k(j): j \in \mathbb{N}\}$ with $\delta(K) \neq 0$ and $\lim _{j} \Delta^{m} X_{k(j)}=X_{0}$.
Let $N_{r}=\left|\left\{k \in I_{r}: k \in K\right\}\right|=\left|K \cap I_{r}\right|$ and $t_{r}=N_{r} / h_{r}$. For any integer $n$ satisfying $k_{r-1}<n \leq k_{r}$, we can write

$$
\begin{aligned}
\frac{1}{n}|\{k \leq n: k \in K\}| & \leq \frac{1}{k_{r-1}}\left|\left\{k \leq k_{r}: k \in K\right\}\right| \\
& =\frac{1}{k_{r-1}}\left\{N_{1}+N_{2}+\cdots+N_{r}\right\} \\
& =\frac{1}{k_{r-1}}\left\{t_{1} h_{1}+t_{2} h_{2}+\cdots t_{r} h_{r}\right\} \\
& =\frac{1}{\sum_{i=1}^{r-1} h_{i}} \sum_{i=1}^{r-1} h_{i} t_{i}+\frac{h_{r}}{k_{r-1}} t_{r} \\
& \leq \frac{1}{\sum_{i=1}^{r-1} h_{i}} \sum_{i=1}^{r-1} h_{i} t_{i}+(H-1) t_{r} .
\end{aligned}
$$

Suppose $t_{r} \rightarrow 0$ as $r \rightarrow \infty$. Since $\theta$ is a lacunary sequence and the first part on the right side of above expression is a regular weighted mean transform of the sequence $t=\left(t_{r}\right)$, therefore it too tends to zero as $r \rightarrow \infty$. Since $n \rightarrow \infty$ as $r \rightarrow \infty$, it follows that $\delta(K)=0$ which is a contradiction as $\delta(K) \neq 0$. Thus $\lim _{r \rightarrow \infty} t_{r} \neq 0$ and therefore $X_{0} \in \Lambda_{S_{\theta}}\left(\Delta^{m}(X)\right)$. Hence $\Lambda_{S}\left(\Delta^{m}(X)\right) \subset \Lambda_{S_{\theta}}\left(\Delta^{m}(X)\right)$.

(iii) This is an immediate consequence of (i) and (ii).

Theorem 14. Let $\theta=\left(k_{r}\right)$ be a lacunary sequence and $X=$ $\left(X_{k}\right)$ a sequence of fuzzy numbers. Then one has the following:

(i) if $\lim \inf q_{r}>1$, then $\Gamma_{S_{\theta}}\left(\Delta^{m}(X)\right) \subset \Gamma_{S}\left(\Delta^{m}(X)\right)$,

(ii) if $\lim \sup q_{r}<\infty$, then $\Gamma_{S}\left(\Delta^{m}(X)\right) \subset \Gamma_{S_{\theta}}\left(\Delta^{m}(X)\right)$,

(iii) if $1<\lim \inf q_{r} \leq \lim \sup q_{r}<\infty$, then $\Gamma_{S}\left(\Delta^{m}(X)\right)=$ $\Gamma_{S_{\theta}}\left(\Delta^{m}(X)\right)$.

Proof. The proof of the theorem can be obtain on the similar lines as that of the above theorem and therefore is omitted here.

Theorem 15. For any lacunary refinement $\theta^{\prime}$ of a lacunary sequence $\theta=\left(k_{r}\right), \Gamma_{S_{\theta}}\left(\Delta^{m}(X)\right) \subseteq \Gamma_{S_{\theta^{\prime}}}\left(\Delta^{m}(X)\right)$ and $\Lambda_{S_{\theta}}\left(\Delta^{m}(X)\right) \subseteq \Lambda_{S_{\theta^{\prime}}}\left(\Delta^{m}(X)\right)$.

Proof. Suppose each $I_{r}$ of $\theta$ contains the points $\left\{k_{r, i}^{\prime}\right\}_{i=1}^{v(r)}$ of $\theta^{\prime}$ so that $k_{r-1}<k_{r, 1}^{\prime}<k_{r, 2}^{\prime}<\cdots<k_{r, v(r)}^{\prime}=k_{r}$, where $I_{r}^{\prime}=\left(k_{r, i-1}^{\prime}, k_{r, i}^{\prime}\right]$. Note that for all $r, v(r) \geq 1$. Let $\left(I_{j}^{*}\right)_{j=1}^{\infty}$ be the sequence of abutting intervals $I_{r, i}^{\prime}$ ordered by increasing right end points. Let $Y_{0} \in \Gamma_{S_{\theta}}\left(\Delta^{m}(X)\right)$, then for each $\epsilon>0$,

$$
\limsup _{r \rightarrow \infty} \frac{1}{h_{r}}\left|\left\{k \in I_{r}: \bar{d}\left(\Delta^{m} X_{k}, Y_{0}\right)<\epsilon\right\}\right|>0 .
$$


As before, write $h_{r, i}^{\prime}=k_{r, i}^{\prime}-k_{r, i-1}^{\prime}$ and $h_{r, 1}^{\prime}=k_{r, 1}^{\prime}-k_{r-1}^{\prime}$. Now for each $\epsilon>0$, we can write

$$
\begin{aligned}
& \frac{1}{h_{r}}\left|\left\{k \in I_{r}: \bar{d}\left(\Delta^{m} X_{k}, Y_{0}\right)<\epsilon\right\}\right| \\
& \quad=\frac{1}{h_{r}} \sum_{I_{j}^{*} \subseteq I_{r}} h_{j}^{*} \frac{1}{h_{j}^{*}}\left|\left\{k \in I_{j}^{*}: \bar{d}\left(\Delta^{m} X_{k}, Y_{0}\right)<\epsilon\right\}\right| \\
& =\frac{1}{h_{r}} \sum_{I_{j}^{*} \subseteq I_{r}} h_{j}^{*}\left(C_{\theta^{\prime}} \chi_{K}\right)_{j},
\end{aligned}
$$

where $\chi_{K}$ is the characteristics function of the set $K=$ $\left\{k \in I_{j}^{*}: \bar{d}\left(\Delta^{m} X_{k}, Y_{0}\right)<\epsilon\right\}$ and $\left(C_{\theta^{\prime}} \chi_{K}\right)_{j}=\left|K \cap I_{j}^{*}\right| / h_{j}^{*}$. Suppose $\lim _{j \rightarrow \infty}\left(C_{\theta^{\prime}} \chi_{K}\right)_{j}=0$. Then the right side of above expression is a regular weighted mean transform of $\left(C_{\theta^{\prime}} \chi_{K}\right)_{j}$ and therefore tends to zero as $j \rightarrow \infty$ which contradicts (36). Thus $\lim _{j \rightarrow \infty}\left(C_{\theta^{\prime}} \chi_{K}\right)_{j} \neq 0$, which shows that $Y_{0} \in$ $\Gamma_{S_{\theta^{\prime}}}\left(\Delta^{m}(X)\right)$. Hence $\Gamma_{S_{\theta}}\left(\Delta^{m}(X)\right) \subseteq \Gamma_{S_{\theta^{\prime}}}\left(\Delta^{m}(X)\right)$.

Similarly, we can prove $\Lambda_{S_{\theta}}\left(\Delta^{m}(X)\right) \subseteq \Lambda_{S_{\theta^{\prime}}}\left(\Delta^{m}(X)\right)$.

\section{Acknowledgment}

The authors are grateful to the referees for their valuable suggestions which improved the readability of the paper.

\section{References}

[1] H. Fast, "Surla convergence statistique," Colloquium Mathematicum, vol. 2, pp. 241-244, 1951.

[2] I. J. Schoenberg, "The integrability of certain functions and related summability methods," American Mathematical Monthly, vol. 66, pp. 361-375, 1951.

[3] J. A. Fridy, "On statistical convergence," Analysis, vol. 5, pp. 301-313, 1985.

[4] J. A. Fridy, "Statistical limit points," Proceedings of the American Mathematical Society, vol. 118, no. 4, pp. 1187-1192, 1993.

[5] J. A. Fridy and C. Orhan, "Statistical limit superior and limit inferior," Proceedings of the American Mathematical Society, vol. 125, no. 12, pp. 3625-3631, 1997.

[6] M. A. Mammadov and S. Pehlivan, "Statistical cluster points and Turnpike theorem in nonconvex problems," Journal of Mathematical Analysis and Applications, vol. 256, no. 2, pp. 686-693, 2001.

[7] J. A. Fridy and C. Orhan, "Lacunary statistical convergent," Pacific Journal of Mathematics, vol. 160, no. 1, pp. 43-51, 1993.

[8] K. Demirci, "On lacunary statistical limit points," Demonstratio Mathematica, vol. 35, pp. 93-101, 2002.

[9] F. Nuray and E. Savaş, "Statistical convergence of sequences of fuzzy numbers," Mathematica Slovaca, vol. 45, pp. 269-273, 1995.

[10] S. Aytar, "Statistical limit points of sequences of fuzzy numbers," Information Sciences, vol. 165, no. 1-2, pp. 129-138, 2004.

[11] S. Aytar and S. Pehlivan, "Statistical cluster and extreme limit points of sequences of fuzzy numbers," Information Sciences, vol. 177, no. 16, pp. 3290-3296, 2007.

[12] S. Aytar, M. A. Mammadov, and S. Pehlivan, "Statistical limit inferior and limit superior for sequences of fuzzy numbers," Fuzzy Sets and Systems, vol. 157, no. 7, pp. 976-985, 2006.
[13] E. Savaş, "On statistically convergent sequences of fuzzy numbers," Information Sciences, vol. 137, no. 1-4, pp. 277-282, 2001.

[14] T. Bilgin, " $\Delta$-statistical and strong $\Delta$-Cesàro convergence of sequences of fuzzy numbers," Mathematical Communications, vol. 8, Article ID 95100, 2003.

[15] M. Işik, "On statistical convergence of generalized difference sequences," Soochow Journal of Mathematics, vol. 30, no. 2, pp. 197-205, 2004.

[16] Y. Altin, M. Et, and R. Çolak, "Lacunary statistical and lacunary strongly convergence of generalized difference sequences of fuzzy numbers," Computers and Mathematics with Applications, vol. 52, no. 6-7, pp. 1011-1020, 2006.

[17] Y. Altin, M. Et, and M. Basarir, "On some generalized difference sequences of fuzzy numbers," Kuwait Journal of Science \& Engineering, vol. 34, no. 1, pp. 1-14, 2007.

[18] H. Altin and R. çolak, "Almost lacunary statistical and strongly almost lacunary convergence of generalized dierence sequences of fuzzy numbers," Journal of Fuzzy Mathematics, vol. 17, no. 4, pp. 951-967, 2009.

[19] R. Çolak, H. Altinok, and M. Et, "Generalized difference sequences of fuzzy numbers," Chaos, Solitons and Fractals, vol. 40, no. 3, pp. 1106-1117, 2009.

[20] M. L. Puri and D. A. Ralescu, "Differential of fuzzy numbers," Journal of Mathematical Analysis and Applications, vol. 91, pp. 552-558, 1983.

[21] F. Nuray, "Lacunary statistical convergence of sequences of fuzzy numbers," Fuzzy Sets and Systems, vol. 99, no. 3, pp. 353355, 1998. 

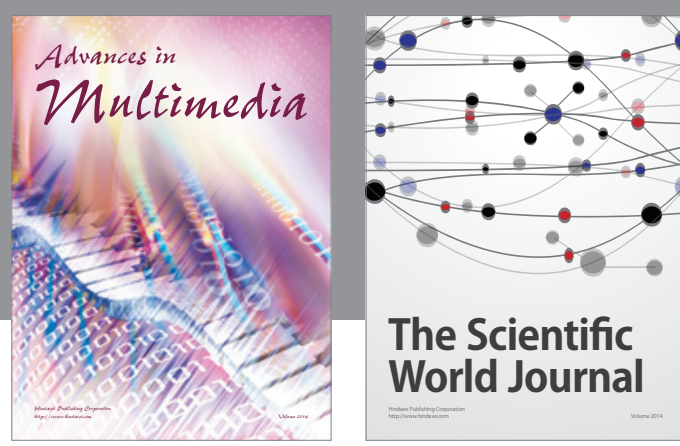

The Scientific World Journal
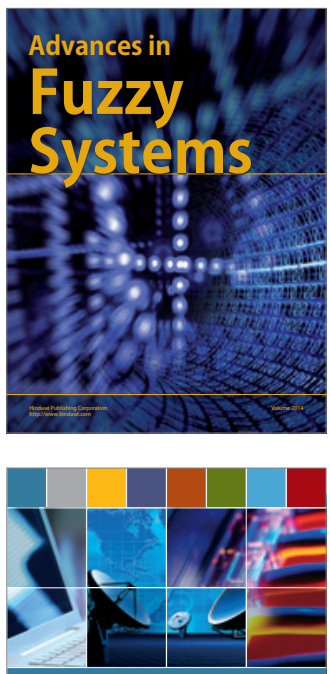

Computer Networks and Communications
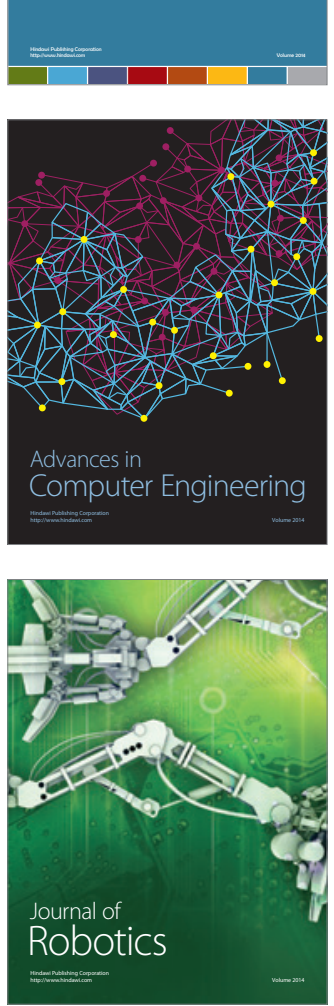
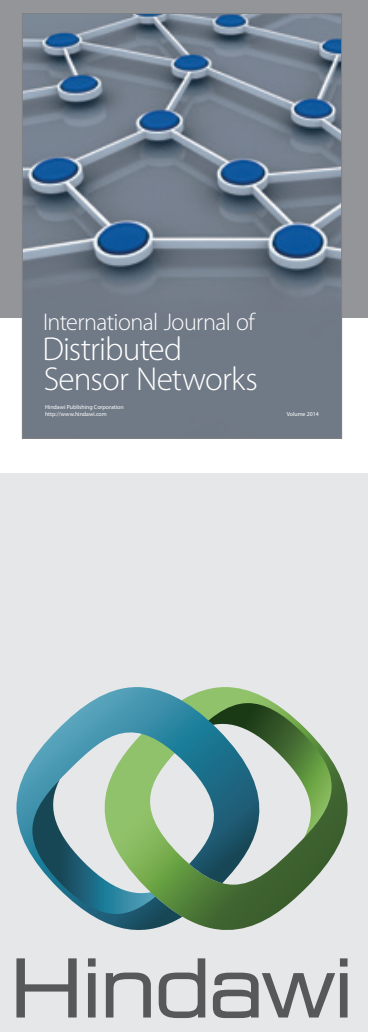

Submit your manuscripts at

http://www.hindawi.com
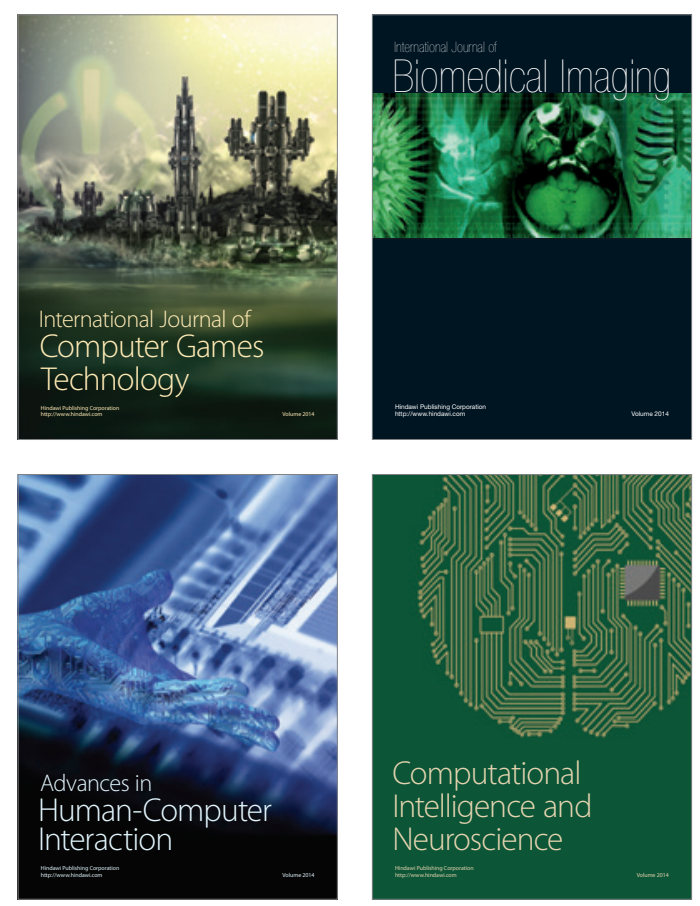
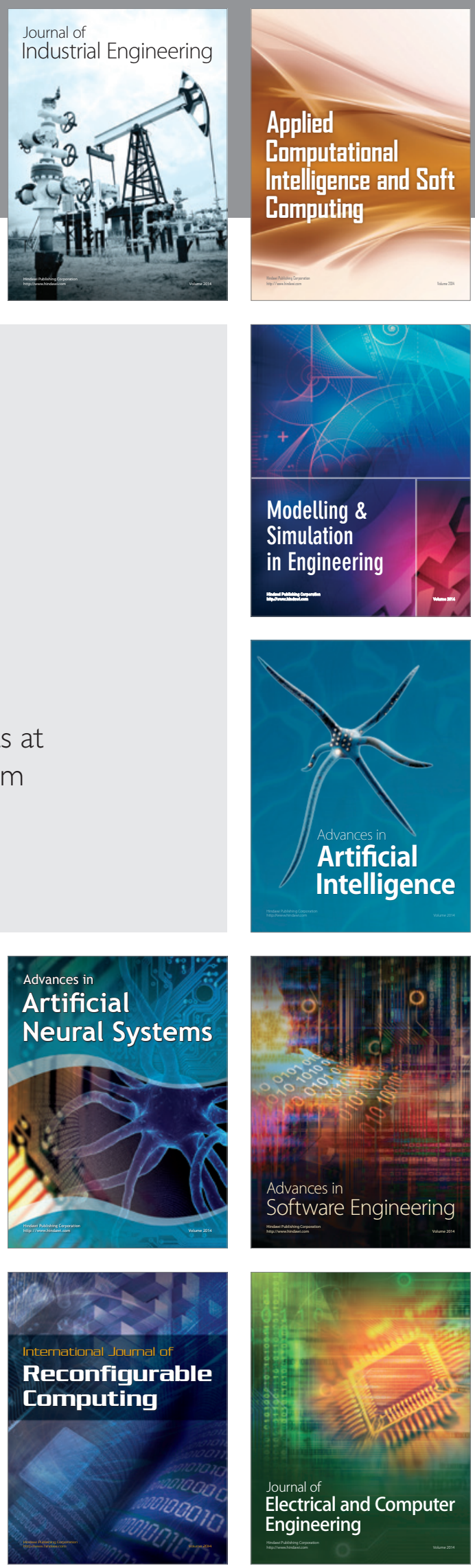